\title{
ÓLEO ESSENCIAL DE LIMÃO NO ENSINO DA CROMATOGRAFIA EM CAMADA DELGADA
}

\author{
Rosaly S. Silva*, Carlos Magno R. Ribeiro, Márcia N. Borges e Giselle S. O. Blois \\ Departamento de Química Orgânica, Instituto de Química, Universidade Federal Fluminense, Campus do Valonguinho, Outeiro
} de S. João Batista, s/n, 24020-141 Niterói - RJ, Brasil

Recebido em 9/1/09; aceito em 15/4/09; publicado na web em 6/10/09

\begin{abstract}
LIME ESSENTIAL OIL IN THE TEACHING OF THIN LAYER CHROMATOGRAPHY. This paper describes a simple experiment employing the essential oil of limes which can be applied in undergraduate organic chemistry laboratory classes for the teaching of thin layer chromatography (TLC). The experiment consists in submit lime peel oil to TLC separation employing hexane and dichloromethane as the eluents and five different systems for visualization of the chromatogram. In one experiment it is possible to teach the different variables of the TLC technique. This experiment may also be performed following vapor distillation and liquidliquid extraction technique in experimental classes.
\end{abstract}

Keywords: lime essential oil; TLC teaching; visualization systems.

\section{INTRODUÇÃO}

A cromatografia em camada delgada (CCD) é um tema importante abordado nos cursos de graduação em química e áreas afins, principalmente no ensino de química orgânica experimental. É uma técnica simples e de execução relativamente rápida. Na química orgânica, a CCD é utilizada, principalmente, como uma ferramenta eficaz de análise qualitativa para avaliação da pureza de uma amostra simples, avaliação do número de componentes de uma mistura, determinação da identidade de uma amostra por comparação com um padrão, identificação de uma ou mais substâncias presentes em uma mistura por comparação com padrões, monitoramento do progresso de uma reação química, escolha de um solvente apropriado para uma separação cromatográfica em coluna e monitoramento de uma separação cromatográfica em coluna.

Nos cursos de Química e áreas afins da Universidade Federal Fluminense (UFF) tem sido observado que o ensino desta técnica deve ser abordado em todas as disciplinas experimentais de química orgânica, para que o aluno consiga compreender e assimilar todas as variáveis envolvidas em uma boa separação cromatográfica.

Diversas experiências para serem utilizadas no ensino da CCD estão descritas na literatura especializada, tanto em livros-texto ${ }^{1}$ quanto em artigos de revistas..$^{2-9}$ Entretanto, na sua grande maioria, apenas uma variável da técnica é estudada como, por exemplo, comparação de fatores de retenção de substâncias diferentes em um mesmo sistema de eluição, ${ }^{2,5,8,9}$ estudo de diferentes sistemas de eluição na separação dos componentes de uma determinada mistura ${ }^{3,4}$ ou estudo de sistemas de revelação diferentes de um cromatograma. ${ }^{6,8}$ Também estão descritas experiências onde há a articulação da CCD com outras técnicas lecionadas nas disciplinas de química orgânica experimental, tais como cromatografia em coluna ${ }^{2,9}$ e extração ácido-base..$^{5,7}$

Dando prosseguimento às nossas pesquisas em ensino de química, ${ }^{10}$ no presente trabalho desenvolvemos uma aula experimental de fácil execução, podendo ser realizada em um período de 3 a 4 h de duração, que tem como tema principal o uso de CCD e suas variáveis, e que também pode ser articulada com outras técnicas de laboratório.

Devido à composição química do óleo essencial obtido de cascas de limão, ${ }^{11}$ que consiste principalmente de monoterpenoides, com uma razoável diversidade de grupos funcionais, é possível, em apenas uma aula experimental, abordar a cromatografia em camada delgada sob

\footnotetext{
*e-mail: gqorosas@vm.uff.br
}

diferentes aspectos, tais como poder de eluição e poder de resolução de solventes, reveladores versus estrutura química e fator de retenção versus estrutura química.

A experiência consiste na cromatografia em camada delgada do óleo essencial de limão, em dois sistemas diferentes de eluição e na visualização do cromatograma em cinco sistemas diferentes de revelação. O óleo essencial de limão pode ser obtido rapidamente através da compressão manual de cascas de limão no início da aula experimental, ou por meio da destilação por arraste a vapor de cascas de limão seguida por extração líquido-líquido, realizadas em uma ou duas aulas anteriores sobre estas técnicas.

\section{PARTE EXPERIMENTAL}

\section{Procedimentos gerais}

Foram utilizados limões taiti, adquiridos em uma feira livre na cidade de Niterói, Rio de Janeiro. Solventes PA Vetec foram utilizados e usados diretamente dos frascos. Foram utilizadas cromatofolhas F254 marca Merck. Lâmpada de UV - Merck modelo E, munida com luz ultravioleta de comprimentos de ondas longo e curto, foi utilizada como revelador, colocando-se as placas cromatográficas sob a incidência de sua luz em caixas escuras adequadas. Soluções de vanilina sulfúrica ${ }^{12}$ e de 2,4-dinitrofenil-hidrazina (DNFH), ${ }^{13}$ preparadas através de metodologia usual, foram utilizadas como reveladores. A aplicação das soluções reveladoras nas placas cromatográficas foi realizada com o uso de pipeta Pasteur, que foi passada horizontalmente ao longo de cada placa. A revelação com iodo foi efetuada colocando-se as placas em cubas de vidro devidamente fechadas contendo cristais de iodo.

$\mathrm{Na}$ aplicação em aula, sugere-se que os procedimentos descritos sejam realizados por equipe de dois alunos e que as soluções reveladoras sejam preparadas antes da aula de CCD.

\section{Extração de óleo por ação mecânica}

Dois limões são descascados cuidadosamente de modo a não permitir a presença de bagaço e suco. As cascas dos limões são manualmente espremidas pela parte externa, obtendo-se algumas gotas de um óleo que são coletadas em um tudo de ensaio de $10 \mathrm{~mL}$ de capacidade munido com um funil simples de $5 \mathrm{~cm}$ de diâmetro. Em 
seguida, o funil é lavado com $2 \mathrm{~mL}$ de diclorometano para o interior do tubo de ensaio (podendo ser utilizado acetato de etila como alternativa), obtendo-se assim o extrato pronto para ser aplicado nas placas cromatográficas.

\section{Extração de óleo por arraste a vapor}

O procedimento de extração descrito a seguir é alternativo e deve ser utilizado no caso de se optar pela articulação do ensino de outras técnicas com a CCD, e poderá ser realizado em uma ou duas aulas anteriores, onde deverão ser abordadas as teorias do arraste a vapor e da extração líquido-líquido.

Dois limões são descascados de modo a não permitir a presença de bagaço e suco nas cascas. Em um balão de $250 \mathrm{~mL}$ são colocadas as cascas dos limões e água é adicionada até o volume final aproximado de $125 \mathrm{~mL}$. Através de uma aparelhagem de destilação simples, adaptada com funil de adição com água, efetua-se o arraste a vapor como codestilação. Destila-se até obter um volume total de aproximadamente $300 \mathrm{~mL}$ de destilado. Para cada $100 \mathrm{~mL}$ de destilado, realiza-se extração com $15 \mathrm{~mL}$ de diclorometano, por cinco vezes (podendo ser utilizado acetato de etila como alternativa). As fases orgânicas são juntadas e, após secagem com sulfato de magnésio anidro e filtração, a solução final é concentrada em um evaporador rotativo, obtendo-se cerca de $1 \mathrm{~mL}$ de óleo, ao qual são adicionados $5 \mathrm{~mL}$ de diclorometano, obtendo-se assim o extrato pronto para ser aplicado nas placas cromatográficas. O óleo deve ser guardado sob refrigeração.

\section{Separação cromatográfica}

As placas cromatográficas são preparadas cortando-se cuidadosamente, com tesoura, tiras de cromatofolha de $2 \times 10 \mathrm{~cm}$. Com o uso de um tubo capilar, uma gota do extrato é aplicada na parte inferior central de cada placa, a aproximadamente $0,5 \mathrm{~cm}$ da borda inferior. Após evaporação do solvente, as placas são submetidas à eluição em uma cuba cromatográfica com hexano. O procedimento é repetido usando-se diclorometano como eluente.

Após a eluição, as placas são observadas sob luz ultravioleta de comprimentos de onda longo e curto e, em seguida, são submetidas à exposição a vapores de iodo. As placas são retiradas da câmara de iodo e assim que as manchas obtidas desaparecem das placas (reversão do complexo com iodo), elas são submetidas à revelação com solução de vanilina sulfúrica ou com solução de 2,4-DNFH. Em cada fase os fatores de retenção, $R f$ s, das manchas observadas são medidos.

\section{RESULTADOS E DISCUSSÃO}

\section{Separação cromatográfica}

Os resultados da cromatografia do óleo das cascas de limão obtido por arraste a vapor, após revelação com os reveladores especificados, encontram-se na Tabela 1. A cromatografia do óleo obtido diretamente por ação mecânica fornece resultados semelhantes.

\section{Constituintes do óleo de limão}

Para uma análise criteriosa dos resultados obtidos, se faz necessário conhecer um pouco da composição do óleo de limão. No catálogo de substâncias químicas Merck Index, ${ }^{14}$ encontra-se uma descrição sucinta dos componentes do óleo essencial de limão. Entretanto, preferimos nos deter no trabalho de Casanova e colaboradores, ${ }^{11}$ por ser um estudo bem detalhado da composição do óleo de limão. Estes pesquisadores analisaram a composição química dos óleos essenciais extraídos de cascas e folhas de limão das espécies Citrus limon (L.) Burm, espécie do limão siciliano, ${ }^{15}$ e Citrus aurantifolia (Christm) Swing., espécie dos limões taiti e galego. ${ }^{15}$ Sobre a espécie a que pertence o limão taiti, utilizado no presente trabalho, eles relatam o estudo de 21 cultivos diferentes de 7 variações botânicas, consideradas como espécies diferentes no sistema Tanaka, tendo sido identificados 62 compostos. ${ }^{11}$ Na maioria das variações botânicas estudadas, os óleos obtidos das cascas eram constituídos quase que exclusivamente de monoterpenoides, sendo a fração de olefinas bem maior que a de substâncias oxigenadas, e o limoneno o principal constituinte de todos os óleos $(39,9-94,4 \%){ }^{11}$ Dentre os terpenoides oxigenados, $\alpha$-terpineol, linalol, acetato de linalila, neral, geranial, acetato de nerila e acetato de geranila estavam presentes em praticamente todas as amostras. A Figura 1 apresenta as estruturas químicas de alguns terpenoides representativos da diversidade de grupos funcionais dos constituintes do óleo de limão.

Através da relação entre os $R f \mathrm{~s}$ (fatores de retenção) das manchas observadas nas placas cromatográficas (Tabela 1) e as estruturas químicas dos possíveis constituintes do óleo obtido (Figura 1), podese estimular uma discussão em aula sobre as variáveis importantes em um processo cromatográfico, citadas na Introdução. A seguir,

Tabela 1. Resultados da cromatografia do óleo de limão extraído por arraste a vapor

\begin{tabular}{|c|c|c|c|c|c|c|c|c|c|c|}
\hline \multirow{2}{*}{$\begin{array}{l}\text { Revelador } \\
\text { Eluente }\end{array}$} & \multicolumn{2}{|c|}{$\begin{array}{c}\text { UV } \\
\text { Ondas longas }\end{array}$} & \multicolumn{2}{|c|}{$\begin{array}{c}\text { UV } \\
\text { Ondas curtas }\end{array}$} & \multicolumn{2}{|c|}{ 2,4-DNFH } & \multicolumn{2}{|c|}{ Vanilina sulfúrica } & \multicolumn{2}{|c|}{ Iodo } \\
\hline & Hexano & $\mathrm{CH}_{2} \mathrm{Cl}_{2}$ & Hexano & $\mathrm{CH}_{2} \mathrm{Cl}_{2}$ & Hexano & $\mathrm{CH}_{2} \mathrm{Cl}_{2}$ & Hexano & $\begin{array}{c}\mathrm{CH}_{2} \mathrm{Cl}_{2} \\
0,79\end{array}$ & Hexano & $\begin{array}{c}\mathrm{CH}_{2} \mathrm{Cl}_{2} \\
0,79\end{array}$ \\
\hline \multirow{9}{*}{$\begin{array}{l}\text { Fator de retenção } \\
(R f)^{\mathrm{a}}\end{array}$} & & & & & & & 0,75 & & 0,75 & \\
\hline & & & & 0,61 & & 0,61 & & 0,61 & & 0,61 \\
\hline & & & & & & & & 0,56 & & 0,56 \\
\hline & & & & 0,50 & & 0,50 & & 0,50 & & 0,50 \\
\hline & & $0,38^{\mathrm{b}}$ & & $0,38^{\mathrm{b}}$ & & & & 0,38 & & 0,38 \\
\hline & & & & & & & & 0,28 & & 0,28 \\
\hline & & & & & & & & 0,08 & & 0,08 \\
\hline & & & 0,02 & & 0,02 & & 0,02 & & & \\
\hline & $0^{\mathrm{b}}$ & & $0^{\mathrm{b}}$ & 0 & 0 & & 0 & 0 & 0 & 0 \\
\hline
\end{tabular}

a erro máximo dos valores de $R f$ é $5 \%$; ${ }^{\text {fluorescente. }}$ 
apresentamos uma análise dos cromatogramas, separando em itens as diversas abordagens que poderão ser tema de discussão.

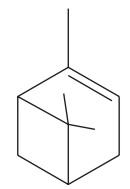

$\alpha$-pineno

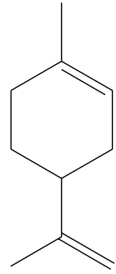

limoneno

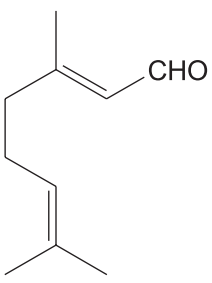

geranial

citral

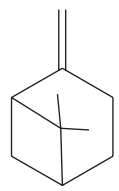

$\beta$-pineno

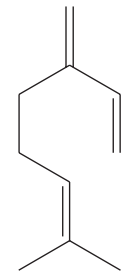

$\beta$-mirceno

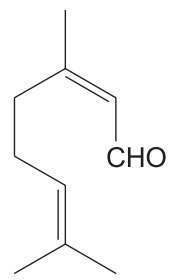

neral,

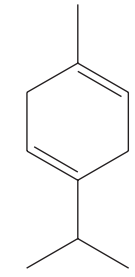

$\gamma$-terpineno

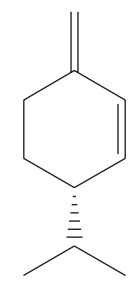

$\beta$-felandreno

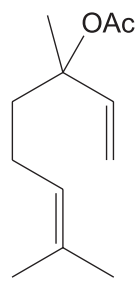

acetato de linalila

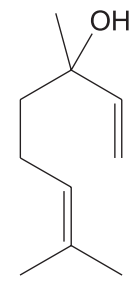

linalol
Figura 1. Estruturas químicas de terpenoides encontrados no óleo essencial de limão

\section{Poder de eluição e resolução}

O maior poder de eluição do diclorometano comparado ao hexano, no adsorvente sílica, é nitidamente observado em todas as placas (Tabela 1). Os $R f$ s são maiores no primeiro solvente. Observa-se, também, uma separação muito melhor dos componentes do óleo de limão com diclorometano do que com hexano. Ou seja, percebe-se o maior poder de resolução do diclorometano para esta mistura. Entretanto, observa-se também que não há separação dos componentes apolares, sendo todos os monoterpenos (hidrocarbonetos com 10 átomos de carbono) eluídos da mesma forma em hexano $(R f 0,75)$ e diclorometano $(R f 0,79)$. A discussão com os alunos pode prosseguir considerando as polaridades da sílica e dos eluentes e a apresentação da série eluotrópica.

\section{Revelação}

Têm-se a oportunidade de comparar a capacidade de revelação de cinco sistemas de revelação diferentes. Observa-se que os reveladores mais gerais, ou seja, aqueles que revelam um grande número de grupos funcionais, são o iodo e a solução de vanilina. A discussão pode prosseguir considerando a utilidade de reveladores específicos para identificação de grupos funcionais específicos, como carbonilas de aldeídos e cetonas com o reagente DNFH, ou a presença de conjugação e de fluorescência, com a observação sob luz UV.

\section{Características estruturais dos componentes das frações obtidas}

As frações obtidas pela separação cromatográfica do extrato de limão, identificadas pelos valores de $R f$ s (Tabela 1 ), podem ser ten- tativamente relacionadas com as características estruturais de seu(s) componente(s), devido, principalmente, ao uso dos diversos reveladores. A Tabela 2 apresenta uma correlação entre os $R f s$ e as características estruturais possivelmente presentes nos componentes das frações.

Da análise da Tabela 2, observa-se que os componentes mais polares, os oxigenados (menores $R f \mathrm{~s}$ ), interagem mais com a sílica do que os apolares, os hidrocarbonetos (maiores $R f \mathrm{~s}$ ), nos dois sistemas de eluição, sendo que a separação entre apolares e polares é maior com o eluente apolar. Observa-se também que os componentes hidrocarbonetos do óleo de limão, apesar da diversidade e de estarem presentes em altas proporções, ${ }^{11}$ não podem ser separados entre si nos sistemas utilizados. Entretanto, os componentes polares apresentam uma grande separação em diclorometano, sendo um fator facilitador para identificar a presença de diversos constituintes no óleo de acordo com a capacidade de revelação em um ou outro revelador, enquanto que em hexano praticamente não se separam.

A utilidade do óleo de limão para o aprendizado da técnica de cromatografia em camada delgada pode ser observada pelos resultados obtidos com alunos da UFF, descritos a seguir.

\section{Aplicação em aula}

Este trabalho foi aplicado em uma aula de Química Orgânica I Experimental com $4 \mathrm{~h}$ de duração para alunos do curso de Química da UFF. Nesta aula foi utilizado o óleo de limão obtido por extração mecânica. Entretanto, poderia ser realizada a destilação por arraste a vapor e extração líquido-líquido em aulas anteriores, e o óleo guardado em geladeira até a aula de $\mathrm{CCD}$, com resultados semelhantes. $\mathrm{O}$ óleo foi submetido à cromatografia em camada delgada conforme relatado na Parte Experimental.

A turma era de doze alunos e foi dividida em seis duplas. Cada dupla preparou duas cromatoplacas. Uma foi eluída em hexano e a outra em diclorometano. Todas as duplas revelaram as duas placas em UV longo e curto. Posteriormente, cada dupla submeteu as mesmas duas placas a uma revelação química, a saber, iodo, vanilina sulfúrica ou 2,4-DNFH, sendo um revelador químico selecionado para cada duas duplas de alunos. Os resultados observados das revelações foram desenhados no quadro, comparados e iniciou-se uma análise dos resultados feita em conjunto. Inicialmente, observou-se que apesar dos alunos já terem feito experiência de CCD no semestre anterior, eles traziam alguns conceitos ou concepções equivocadas. As principais estavam relacionadas ao desconhecimento de reveladores diferentes da lâmpada de UV e ao fato de acharem que a revelação de uma única mancha assegurava a pureza da amostra analisada. Com o experimento realizado eles reformularam esses conceitos e verificaram que a escolha dos eluentes está relacionada com a resolução de uma mistura e os fatores de retenção observados. Puderam verificar que a revelação de uma substância depende de características de sua estrutura química, ou da presença de determinados grupos funcionais, e do tipo de revelador usado.

Os alunos avaliaram de forma positiva o experimento. Acharam de fácil compreensão e bastante instrutivo em relação aos principais fundamentos da CCD. A mudança de atitude dos alunos pôde ser observada quando, durante o desenvolvimento do projeto final da disciplina (como forma de avaliação, os alunos desenvolvem um pequeno projeto onde utilizam as técnicas aprendidas), todos tiveram a preocupação de utilizar mais de um tipo de eluente e de revelador na CCD das amostras analisadas.

\section{CONCLUSÕES}

Neste trabalho foi possível demonstrar que uma experiência simples, empregando um alimento de uso cotidiano, o limão, uma fruta bem conhecida, pode ser usada com bons resultados no ensino da cromatografia 
Tabela 2. Atribuição de características estruturais aos componentes das frações do óleo de limão cromatografado por eluição com hexano e diclorometano

\begin{tabular}{|c|c|c|c|c|c|}
\hline \multicolumn{3}{|c|}{ Eluição em hexano } & \multicolumn{3}{|c|}{ Eluição em diclorometano } \\
\hline $\mathrm{Rf}$ & Componentes prováveis & revelação & $\mathrm{Rf}$ & Componentes prováveis & revelação \\
\hline & & & 0,79 & hidrocarbonetos & vanilina, iodo \\
\hline \multirow[t]{7}{*}{0,75} & hidrocarbonetos & vanilina, iodo & & & \\
\hline & & & 0,61 & $\begin{array}{l}\text { oxigenados incluindo } \\
\text { aldeídos e conjugados }\end{array}$ & $\begin{array}{l}\text { vanilina, iodo, } \\
\text { 2,4-DNFH, } \\
\text { UV curto }\end{array}$ \\
\hline & & & 0,56 & oxigenados não conjugados & vanilina, iodo \\
\hline & & & 0,50 & $\begin{array}{l}\text { oxigenados incluindo } \\
\text { aldeídos e conjugados }\end{array}$ & $\begin{array}{l}\text { vanilina, iodo, } \\
\text { 2,4-DNFH, } \\
\text { UV curto }\end{array}$ \\
\hline & & & 0,38 & $\begin{array}{l}\text { oxigenados incluindo } \\
\text { conjugados e/ou fenois }\end{array}$ & $\begin{array}{l}\text { vanilina, iodo, } \\
\text { UV curto, UV longo }\end{array}$ \\
\hline & & & 0,28 & oxigenados não conjugados & vanilina, iodo \\
\hline & & & 0,08 & $\begin{array}{l}\text { oxigenados incluindo } \\
\text { conjugados e/ou fenois }\end{array}$ & $\begin{array}{l}\text { vanilina, iodo, } \\
\text { UV curto, UV longo }\end{array}$ \\
\hline 0,02 & $\begin{array}{l}\text { oxigenados incluindo } \\
\text { aldeídos e conjugados }\end{array}$ & $\begin{array}{l}\text { vanilina, 2,4-DNFH, } \\
\text { UV curto }\end{array}$ & & & \\
\hline 0 & $\begin{array}{l}\text { oxigenados incluindo } \\
\text { aldeídos, conjugados } \\
\text { e/ou fenois }\end{array}$ & $\begin{array}{c}\text { vanilina, iodo, } \\
\text { 2,4-DNFH, } \\
\text { UV curto, UV longo }\end{array}$ & 0 & oxigenados & $\begin{array}{l}\text { vanilina, iodo, } \\
\text { UV curto }\end{array}$ \\
\hline
\end{tabular}

Obs.: o termo conjugados é empregado como referência à presença de carbonila conjugada e/ou anel aromático.

em camada delgada (CCD). O uso de dois eluentes e cinco sistemas de revelação diferentes possibilitou a separação dos constituintes do óleo de limão em diversas frações, de acordo com as características estruturais das substâncias presentes. Com esta experiência, os alunos de uma turma da UFF foram apresentados, ao mesmo tempo e de forma articulada, aos princípios da $\mathrm{CCD}$, tendo sido observado que o aprendizado pelos alunos foi mais rápido e consistente do que anteriormente verificado com outras experiências. O ensino de CCD, como descrito na aula proposta, também pode ser combinado com o ensino de outras técnicas, tais como destilação por arraste a vapor e extração líquido-líquido.

\section{AGRADECIMENTOS}

A S. J. Garden, M. C. Saraiva de Mattos e L. M. Viana pela colaboração na realização deste trabalho.

\section{REFERÊNCIAS}

1. Pavia, D. L.; Lampman, G. M.; Kriz, G. S.; Engel, R. G.; Introduction to Organic Laboratory Techniques, A Microscale Approach, $3^{\text {rd }}$ ed., Saunders: Orlando, 1999; Gilbert, J. C.; Martin, S. F.; Experimental Organic Chemistry: a miniscale and microscale approach, $2^{\text {nd }}$ ed., Saunders: Orlando, 1998; Collins, C. H.; Braga, G. L.; Bonato, P. S.; Introdução a Métodos Cromatográficos, 7ª ed., Unicamp: Campinas, 1997; Moore, J. A.; Dalrymple, D. L.; Rodig, O. R.; Experimental Methods in Organic Chemistry, $3^{\text {rd }}$ ed., Saunders: Orlando, 1982.
2. Reynolds, R. C.; O’Dell, C. A.; J. Chem. Educ. 1992, 12, 989.

3. Scim, A. J.; J. Chem. Educ. 1985, 62, 361.

4. Olesen, B.; Hopson, D.; J. Chem. Educ. 1983, 60, 232.

5. McKone, H. T.; Nelson, G. J.; J. Chem. Educ. 1976, 53, 722.

6. Chasar, D. W.; Toth, G. B.; J. Chem. Educ. 1974, 51, 22.

7. Chasar, D. W.; Toth, G. B.; J. Chem. Educ. 1974, 51, 487.

8. Madsen, B. C.; J. Chem. Educ. 1973, 50, 853.

9. Ruppel, I. B., Jr.; Cuneo, F. L.; Krause, J. G.; J. Chem. Educ. 1971, 48, 635.

10. Borges, M. N.; Ribeiro, C. M. R.; Souza, N. A.; Educ. Química 2005, 16, 586; Ribeiro, C. M. R. Em Contribuições aos Professores de Química do Ensino Médio; Coutinho, L. G. R.; Ferreira, V. F., eds.; UFF: Niterói, 2005, cap. 16; Souza, N. A.; Ribeiro, C. M. R., Quim. Nova 2007, 30, 1026.

11. Francheschi, E.; Grings, M. B.; Frizzo, C. D.; Oliveira, J. V.; Dariva, C.; Fluid Phase Equilib. 2004, 226, 1; Lota, M.-L.; Serra, D.de R.; Tomi, F.; Jacquemond, C.; Casanova, J.; J. Agric. Food Chem. 2002, 50, 796.

12. Marques, J. A.; Borges, C. P. F.; Práticas de Química Orgânicas, Átomo: Campinas, 2007.

13. Shriner, R. L.; Herman, C. K. F.; Morril, T. C.; Curtin, D. Y., Fuson, R. C.; The Systematic Identification of Organic Compounds, $7^{\text {th }}$ ed., Wiley: New York, 1998.

14. Windholz, M.; The Merck Index: an Encyclopedia of Chemicals and Drugs, $9^{\text {th }}$ ed., Merck: Rahway, 1976.

15. Lorenzi, H.; Matos, F. J. A.; Plantas Medicinais no Brasil: nativas e exóticas, Instituto Plantarum: Nova Odessa, 2002. 